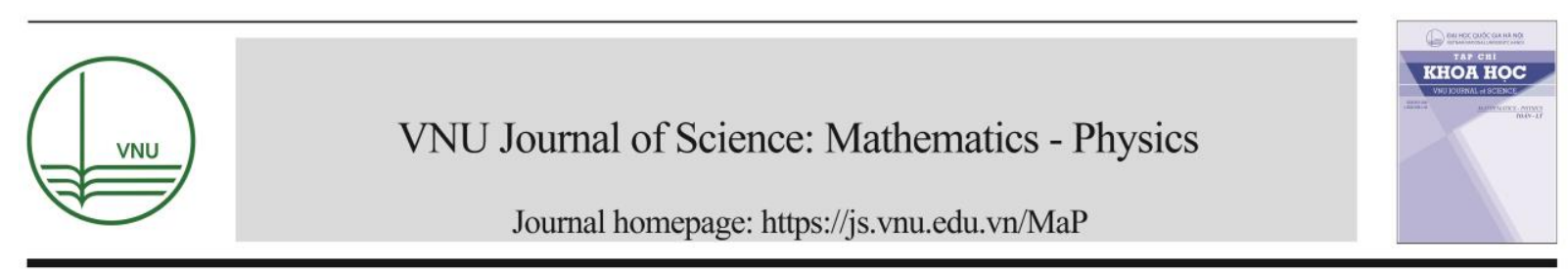

\title{
The Zeta Potential Calculation for Fluid Saturated Porous Media Using Linearized and Nonlinear Solutions of Poisson-Boltzmann Equation
}

\author{
Luong Duy Thanh* \\ Thuy Loi University, 175 Tay Son, Dong Da, Hanoi, Vietnam
}

Received 25 January 2018, Accepted 28 March 2018

\begin{abstract}
Theoretical models have been developed to calculate the zeta potential based on the solution of the linearized approximation of the Poisson-Boltzmann equation (PB). The approximation is only valid for the small magnitude of the surface potential. However, the surface potential available in published experimental data normally does not satisfy that condition. Therefore, the complete analytical solution to the PB equation (nonlinear equation) needs to be considered. In this work, the comparison between the linearized and nonlinear solutions has been performed. The results show that the linearized solution always overestimates the absolute value of the electric potential in the electric double layer as well as the zeta potential. For a small magnitude of the surface potential $\left(\left|\varphi_{d}\right| \leq 25 \mathrm{mV}\right)$, the electric potential distribution predicted from the linearized solution is almost the same as that predicted from the nonlinear solution. It is also shown that the zeta potential computed from the linearized PB solution closely matches with that computed from the nonlinear solution for the fluid $\mathrm{pH}=5-8$ and the shear plane distance of $2.4 \times 10^{-10} \mathrm{~m}$. Therefore, the solution of the linearized PB equation can be used to calculate the zeta potential under that condition. This is validated by comparing the linearized and nonlinear solutions with experimental data in literature.
\end{abstract}

Keywords: Zeta potential, porous media, electric double layer, Poisson-Boltzmann equation.

\section{Introduction}

The electrokinetic phenomena are induced by the relative motion between the fluid and the solid surface. In a porous medium such as rocks or soils, the electric current density, linked to the ions within the fluid, is coupled to the fluid flow and that coupling is called electrokinetics e.g. [1]. Measurement of the electrokinetics in porous media is becoming increasingly more important in

\footnotetext{
* Tel.: 84-936946975.

Email: luongduythanh2003@yahoo.com

https//doi.org/ 10.25073/2588-1124/vnumap.4259
} 
geophysical applications. For example, it could be used to map subsurface flow and detect subsurface flow patterns in oil reservoirs [e.g., 2- 6], geothermal areas and volcanoes [e.g.,7, 8, 9], detection of contaminant plumes [e.g., 10, 11]. It has also been proposed to use the monitoring of electrokinetics to detect at distance the propagation of a water front in a reservoir [e.g., 12] or to predict earthquakes [e.g., 13].

The zeta potential of a solid-liquid interface is one of the most important parameters in electrokinetics. Theoretical models have been developed to calculate the zeta potential based on the solution of the linearized approximation of the PB equation for the electric double layer [e.g., 14, 15]. The approximation is only valid for the small magnitude of the surface potential $\left(\left|\varphi_{d}\right| \leq 25 \mathrm{mV}\right)[16$, 17]. However, the surface potential available in published experimental data normally does not satisfy that condition. Therefore, a complete analytical solution to the nonlinear PB equation needs to be considered. Additionally, to the best of my knowledge the difference in the zeta potential calculation between the solutions of the linearized and nonlinear PB equation has not yet been evaluated. In this work, the comparison between the linearized and nonlinear solutions has been performed for silica surfaces because of the availability of input parameters for the model as well as experimental data in literature [e.g., 14, 15]. It is found that the linearized solution always overestimates the absolute value of the electric potential in the electric double layer (EDL) as well as the zeta potential. For a small magnitude of the surface potential $\left(\left|\varphi_{d}\right| \leq 25 \mathrm{mV}\right)$, the linearized PB solution could be used to predict the electric potential distribution in the EDL instead of the more complicated nonlinear PB solution. The results also show that at a given electrolyte concentration, the zeta potential computed from the linearized PB solution closely matches with that computed from the nonlinear solution for the fluid $\mathrm{pH}$ $=5-8$ that are normally encountered in published experimental data and the shear plane distance of $2.4 \times 10^{-10} \mathrm{~m}$. Therefore, the solution of the nonlinear PB equation can be used to calculate the zeta potential under that condition. This is validated by comparing the linearized and nonlinear solutions with each other and with experimental data in literature. It should be noted that if the shear distance is taken as $2.4 \times 10^{-9} \mathrm{~m}$ or larger value and the fluid $\mathrm{pH}$ is larger than 8 , one needs to use the linearized PB solution to calculate the zeta potential.

\section{Theoretical background of the zeta potential}

\subsection{Physical chemistry of the electric double layer}

Solid grain surfaces of the rocks immersed in aqueous systems acquire a surface electric charge, mainly via the dissociation of silanol groups - $\mathrm{SiOH}^{0}$ (where the superscript "0" means zero charge) and the adsorption of cations on solid surfaces. The reactions at a solid silica surface (silica is the main component of rocks) in contact with fluids have been well described in literature [e.g, 14, 15, 18]. The reactions at the silanol surfaces in contact with 1:1 electrolyte solutions are:

$\mathrm{SiOH}^{0} \Leftrightarrow>\mathrm{SiO}^{-}+\mathrm{H}^{+}$,

for deprotonation of silanol groups

and

$\mathrm{SiOH}^{0}+\mathrm{Me}^{+} \Leftrightarrow \mathrm{SiOMe}^{0}+\mathrm{H}^{+}$,

for cation adsorption on silica surfaces $\left(\mathrm{Me}^{+}\right.$refer to monovalent cations in the electrolytes such as $\mathrm{K}^{+}$or $\mathrm{Na}^{+}$). It should be noted that further protonation of the silanol surfaces is expected only under extremely acidic conditions $(\mathrm{pH}<2-3)$ and is not considered. Similarly, the protonation of doubly 
coordinated groups $\left(\mathrm{Si}_{2} \mathrm{O}^{0}\right)$ is not taken into account because these are normally considered inert $[14$, $15,18]$.

According to $[14,15]$, the disassociation constant for deprotonation of the silica surfaces is determined as

$$
K_{(-)}=\frac{\Gamma_{S i O^{-}}^{0} . \alpha_{H^{+}}^{0}}{\Gamma_{S i O H}^{0}},
$$

and the binding constant for cation adsorption on the silica surfaces is determined as

$$
K_{\mathrm{Me}}=\frac{\Gamma_{\mathrm{SiOMe}}^{0} \cdot \alpha_{\mathrm{H}^{+}}^{0}}{\Gamma_{\mathrm{SiOH}}^{0} \cdot \alpha_{\mathrm{Me}^{+}}^{0}},
$$

where $\Gamma_{i}^{0}$ is the surface site density of surface species $i\left(\operatorname{sites} / \mathrm{m}^{2}\right)$ and $\alpha_{i}^{0}$ is the activity of an ionic species $i$ at the closest approach of the mineral surface (no units).

The total density of surface sites $\left(\Gamma_{S}^{0}\right)$ is determined as follows

$$
\Gamma_{S}^{0}=\Gamma_{S i O H}^{0}+\Gamma_{S i O^{-}}^{0}+\Gamma_{\text {SiOMe }}^{0}
$$

The mineral surface charge density $Q_{S}^{0}$ in $\mathrm{C} / \mathrm{m}^{2}$ can be found by

$$
Q_{S}^{0}=-e . \Gamma_{S_{i O^{-}}}^{0},
$$

where $e$ is the elementary charge.

Due to a charged solid surface, an electric double layer (EDL) is developed at the liquid-solid interface when solid grains of rocks are in contact with the liquid. The EDL is made up of (1) the Stern layer where cations are adsorbed on the surface and are immobile due to the strong electrostatic attraction and (2) the diffuse layer where the number of cations exceeds the number of anions and the ions are mobile. The closest plane to the solid surface in the diffuse layer at which flow occurs is termed the shear plane and the electric potential at this plane is called the zeta potential $(\zeta)$.

\subsection{Electric potential distribution in the EDL}

Following assumptions are used in the EDL theory [e.g., 19, 20]: (1) ions in the double layer are considered as point charges and there are no chemical interactions between them; (2) charges on the solid grain surface are uniformly distributed; (3) the solid surface is a flat plate that is large relative to thickness of double layer and (4) the dielectric constant of the medium is the same everywhere in the liquid.

In the EDL theory, the local concentrations of cations, $C^{+}(x)$ and of anions, $C^{-}(x)\left(\mathrm{mol} \mathrm{m}^{-3}\right)$ in the liquid in the pore space at distance $x$ from the solid surface are expressed as functions of the electric potential $\psi(\mathrm{x})$. According to Boltzmann theorem [e.g., 21, 22], one has

$C^{+}(x)=C_{b}^{+} e^{\frac{-e Z \psi(x)}{k_{b} T}}$

and

$$
C^{-}(x)=C_{b}^{-} e^{\frac{e Z \psi(x)}{k_{b} T}}
$$


where $C_{b}^{+}$and $C_{b}^{-}$are concentration of the cations and concentration of the anions, respectively at large distance from the solid surface where the electric potential is zero $(\psi(\infty)=0), Z$ is the valence of the ions under consideration (dimensionless); $k_{b}$ is the Boltzmann's constant $\left(1.38 \times 10^{-23} \mathrm{~J} / \mathrm{K}\right), T$ is temperature (in $\mathrm{K}$ ).

The Poisson equation relating the electric potential, $\psi(x)$ (in V) and volumetric charge density, $\rho(x)$ (in $\mathrm{C} \mathrm{m}^{-3}$ ) in the liquid is expressed as [e.g., 21, 22]

$$
\frac{d^{2} \psi(x)}{d x^{2}}=-\frac{\rho(x)}{\varepsilon_{r} \varepsilon_{0}}
$$

where $\varepsilon_{r}$ is the relative permittivity of the fluid $\left(78.5\right.$ at $25^{\circ} \mathrm{C}$ for water), $\varepsilon_{o}$ is the dielectric permittivity in vacuum $\left(8.854 \times 10^{-12} \mathrm{C}^{2} \mathrm{~J}^{-1} \mathrm{~m}^{-1}\right)$.

For single type of ions in the liquid, $\rho(x)$ is given by [e.g., 20]

$$
\rho(x)=N e Z\left[C^{+}(x)-C^{-}(x)\right]=N e Z C_{b}\left(e^{-\frac{e Z \psi(x)}{k_{b} T}}-e^{\frac{e Z \psi(x)}{k_{b} T}}\right)
$$

where $C_{b}=C_{b}^{+}=C_{b}^{-}$for symmetric electrolytes such as $\mathrm{NaCl}$ or $\mathrm{CaSO}_{4}$ representing number of ions (anion or cation) expressed in mole per unit volume $\left(\mathrm{mol} \mathrm{m}^{-3}\right), e$ is the elementary charge $(\mathrm{e}=$ $\left.1.6 \times 10^{-19} \mathrm{C}\right)$ and $N$ is the Avogadro's number $\left(6.022 \times 10^{23} / \mathrm{mol}\right)$.

Putting Eq. (10) into Eq. (9), one obtains

$$
\frac{d^{2} \psi(x)}{d x^{2}}=-\frac{N e Z C_{b}}{\varepsilon_{r} \varepsilon_{0}}\left(e^{-\frac{e Z \psi(x)}{k_{b} T}}-e^{\frac{e Z \psi(x)}{k_{b} T}}\right)
$$

or

$$
\frac{d^{2} \psi(x)}{d x^{2}}=\frac{2 N e Z C_{b}}{\varepsilon_{r} \varepsilon_{0}} \sinh \left(\frac{e Z \psi(x)}{k_{b} T}\right)
$$

Eq. (12) is known as the PB equation. The boundary conditions to be satisfied for flat solid surfaces are: (1) the potential at the surface $x=0, \psi(0)=\varphi_{d}$ that is called the surface potential or Stern potential); (2) the potential in the bulk liquid at distance $x=\infty, \psi(\infty)=0$ and $\left.\frac{d \psi(x)}{d x}\right|_{x=\infty}=0$ [e.g., 20].

a) Linearized solution of Poisson-Boltzmann equation

It is seen that if $\left|\frac{e Z \varphi_{d}}{k_{b} T}\right|<<1\left(\left|\varphi_{d}\right| \leq 25 \mathrm{mV}\right.$ for $Z=1$ at $\left.25^{\circ} \mathrm{C}\right)$, then $\sinh \left(\frac{e Z \psi(x)}{k_{b} T}\right) \approx \frac{e Z \psi(x)}{k_{b} T}$ [e.g., 16, 17, 23, 24]. Therefore, Eq. (12) linearizes as follows

$$
\frac{d^{2} \psi(x)}{d x^{2}}=\frac{2 N e^{2} Z^{2} C_{b}}{\varepsilon_{r} \varepsilon_{0} k_{b} T} \psi(x)
$$

The solution to linearized $\mathrm{PB}$ equation satisfying the boundary conditions is given by [e.g., 25] 
$\psi(x)=\varphi_{d} \exp \left(-\frac{x}{\chi_{d}}\right)$

where $\chi_{d}$ is called Debye length given by $\chi_{d}=\sqrt{\frac{\varepsilon_{o} \varepsilon_{r} k_{b} T}{2 N e^{2} Z^{2} C_{b}}}\left(C_{b}\right.$ in mol m $\left.{ }^{-3}\right)$. If $C_{b}$ is in mol $\mathrm{L}^{-1}$, then $\chi_{d}=\sqrt{\frac{\varepsilon_{o} \varepsilon_{r} k_{b} T}{2000 N e^{2} Z^{2} C_{b}}}$.

b) Nonlinear solution of Poisson-Boltzmann equation

The exact solution to PB equation - Eq. (12) for single type of ions has been found in both [20] and [26]. However, the solution presented in [20] has a more simplified form as below:

$$
\psi(x)=\frac{2 k_{b} T}{e Z} \ln \left(\frac{1-A \exp \left(-\frac{x}{\chi_{d}}\right)}{1+A \exp \left(-\frac{x}{\chi_{d}}\right)}\right)
$$

where $A=\frac{1-\exp \left(\frac{e Z}{2 k_{b} T} \varphi_{d}\right)}{1+\exp \left(\frac{e Z}{2 k_{b} T} \varphi_{d}\right)}$

Therefore, Eq. (15) is used as the exact solution to nonlinear PB equation to calculate the zeta potential in this work.

\subsection{The surface potential and zeta potential}

In a theoretical model that has been well described in [e.g., 14, 15], the surface electric potential $\varphi_{d}$ for a solid surface in contact with 1:1 electrolytes $(Z=1)$ is given by

$$
\varphi_{d}=\frac{2 k_{b} T}{3 e} \ln \left\{\frac{\sqrt{8.10^{3} \varepsilon_{o} \varepsilon_{r} k_{b} T N}\left(10^{-p H}+K_{M e} C_{b}\right)}{2 e \Gamma_{S}^{0} K_{(-)}}\left[\frac{C_{b}+10^{-p H}+10^{p H-p K_{w}}}{\sqrt{C_{b}}}\right]\right\}
$$

where $p H$ is the fluid $\mathrm{pH}$ and $K_{w}$ is the disassociation constant of water.

According to the definition, the zeta potential is the electric potential at the shear plane. Therefore, one has

$$
\zeta=\left.\psi(x)\right|_{x=\chi_{\varsigma}}
$$

where $\psi(x)$ is the electric potential distribution in the EDL given by Eq (14) or Eq. (15), $\chi_{\zeta}$ is the shear plane distance (the distance from the solid surface to the shear plane). There is currently no method to evaluate the shear plane distance. There are few independent reports of the shear plane distance. For example, the shear plane distance $\chi_{\zeta}$ is found to be $2.4 \times 10^{-10} \mathrm{~m}$ in [15] but $2.0 \times 10^{-9} \mathrm{~m}$ in [27]. 


\section{Results and discussion}

A system of 1:1 symmetric electrolytes (e.g., $\mathrm{NaCl}, \mathrm{KNO}_{3}$ ) and silica solid surfaces are considered for the modeling in this work because of the availability of input parameters for the model as well as experimental data in literature [e.g., 14, 15]. Therefore, the valence $Z=1$ is used from Eq. (7) to Eq. (15).

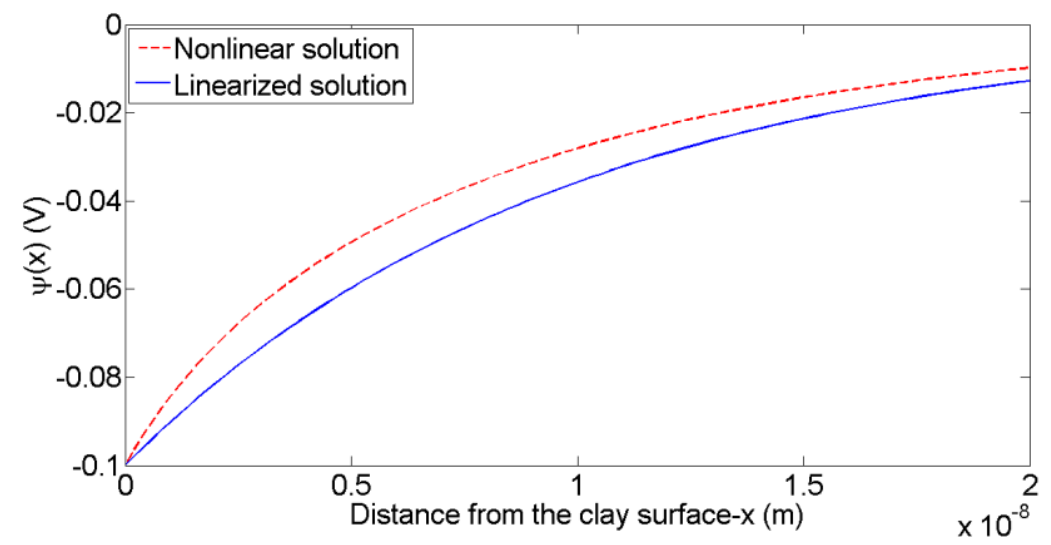

(a) $\varphi_{d}=-0.1 \mathrm{~V} ; \mathrm{C}_{\mathrm{b}}=10^{-3} \mathrm{~mol} / \mathrm{L}$

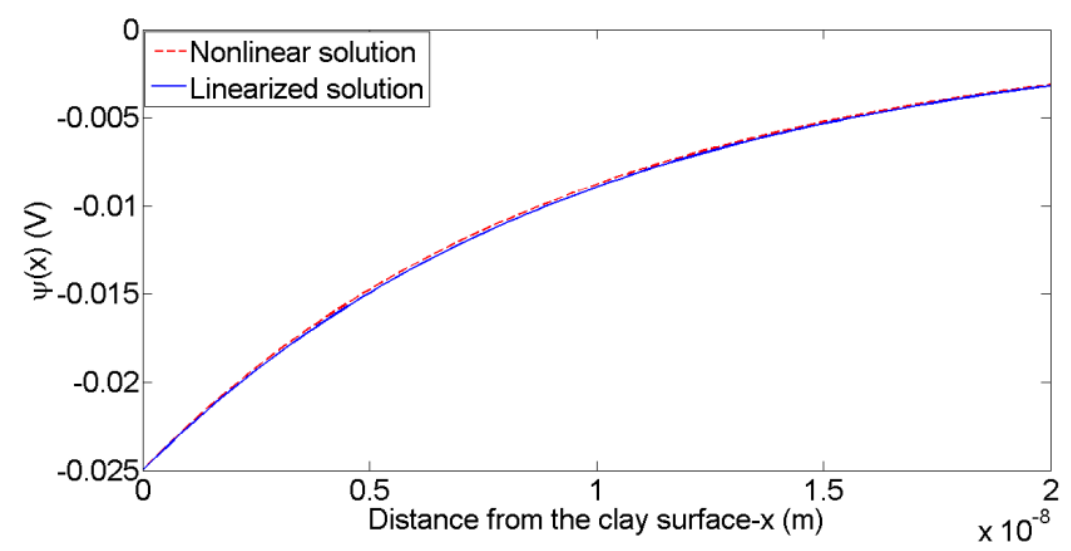

(b) $\varphi_{d}=-0.025 \mathrm{~V} ; \mathrm{C}_{\mathrm{b}}=10^{-3} \mathrm{~mol} / \mathrm{L}$

Figure 1. The variations of the electric potential with respect to distance $x$ from the solid surface computed using the solutions of the linearized and nonlinear PB equation.

\subsection{The distribution of $\psi(x)$}

The variation of the electric potential $\psi(x)$ with respect to distance $x$ from the solid surface predicted from the linearized and nonlinear solutions (Eq. (14) and Eq. (15), respectively) for two different values of the surface potential $\left(\varphi_{d}=-0.1 \mathrm{~V}\right.$ and $\left.\varphi_{d}=-0.025 \mathrm{~V}\right)$ is shown in Fig. 1 (electrolyte concentration $C_{b}$ is taken to be $10^{-3} \mathrm{M}$ ). It is seen that the solution of linearized $\mathrm{PB}$ 
equation overestimates the absolute value of the electric potential for $\varphi_{d}=-0.1 \mathrm{~V}$ as shown in Fig. 1(a). For the smaller absolute value of the surface potential $\varphi_{d}=-0.025 \mathrm{~V}$, the prediction from the nonlinear and linearized solutions is almost the same as shown in Fig. 1(b). It is inferred that the difference in the electric potential distribution predicted by the two solutions increases with increasing absolute value of the surface potential. For a small magnitude of the surface potential $\left(\left|\varphi_{d}\right| \leq 25 \mathrm{mV}\right)$, the linearized PB solution could be used to predict the electric potential distribution in the EDL as expected in literature [e.g., 16, 17]. The variation of $\psi(x)$ with distance $x$ for two different electrolyte concentrations $\left(C_{b}=10^{-2} \mathrm{M}\right.$ and $\left.C_{b}=10^{-3} \mathrm{M}\right)$ is also shown in Fig. 2 . It is seen that the deviation of the electric potential obtained by the nonlinear and linearized solutions is more for lower the electrolyte concentration.

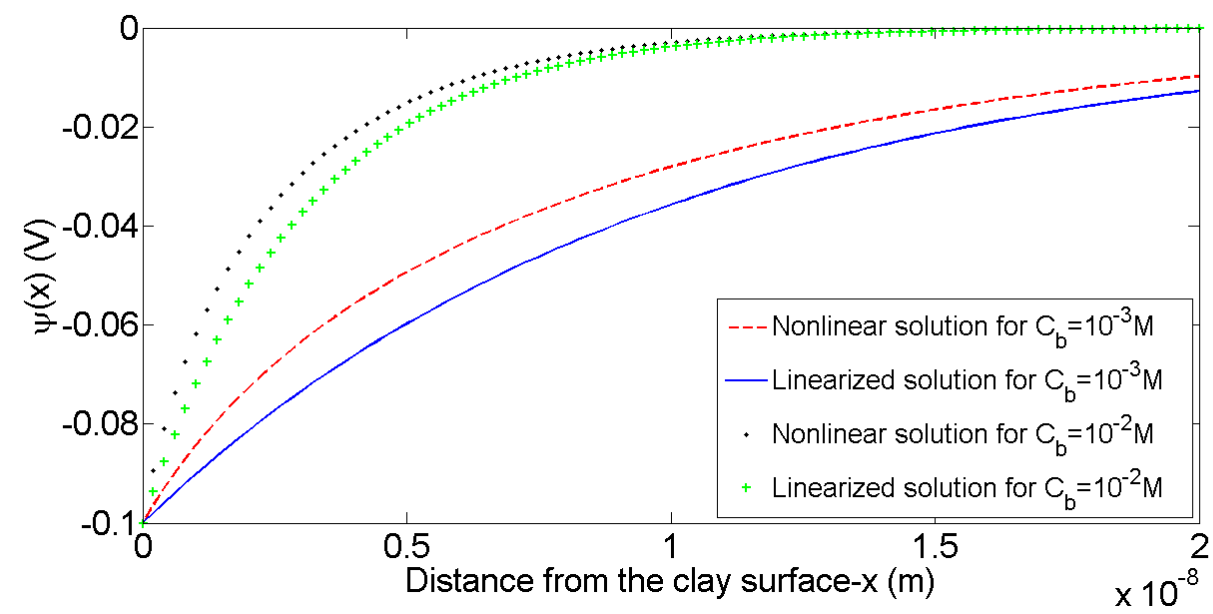

Figure 2. The variations of the electric potential with respect to distance $x$ using the solutions of the linearized and nonlinear PB equation for two different electrolyte concentrations.

\subsection{The zeta potential comparison}

To evaluate the variation of the zeta potential with respect the electrolyte concentration from both the linearized and nonlinear solutions of the PB equation, one need to calculate the surface potential from Eq. (16). Input parameters that are available in [14, 15] for silica are used for Eq. (16). Namely, the value of the disassociation constant $K_{(-)}$is taken as $10^{-7.1}$. The surface site density $\Gamma_{S}^{0}$ is taken as $10 \times 10^{18} \mathrm{site} / \mathrm{m}^{2}$. The disassociation constant of water $K_{w}$ is taken as $9.214 \times 10^{-15}$ at $25^{\circ} \mathrm{C}\left(p K_{w}=-\right.$ $\left.\log _{10}\left(K_{w}\right)\right)$. The fluid $\mathrm{pH}$ is taken as 7 . The binding constant for cation adsorption of $\mathrm{Na}^{+}$on silica surface $K_{\mathrm{Me}}\left(\mathrm{Na}^{+}\right)$is taken as $10^{-7.5}$. The shear plane distance $\chi_{\zeta}$ is taken as $2.4 \times 10^{-10} \mathrm{~m}$ and $2.4 \times 10^{-9}$ $\mathrm{m}$ for comparison.

Fig. 3 shows the variations of the zeta potential with electrolyte concentration using the solutions of the linearized and nonlinear PB equation. It is found that the zeta potential in magnitude predicted from the linearized solution is significantly larger (up to 15\%) than that predicted from the nonlinear solution in the studied range of electrolyte concentration for the shear plane distance of $2.4 \times 10^{-9} \mathrm{~m}$. However, a slight difference in the zeta potential is observed for the shear plane distance of $2.4 \times 10^{-10} \mathrm{~m}$. 


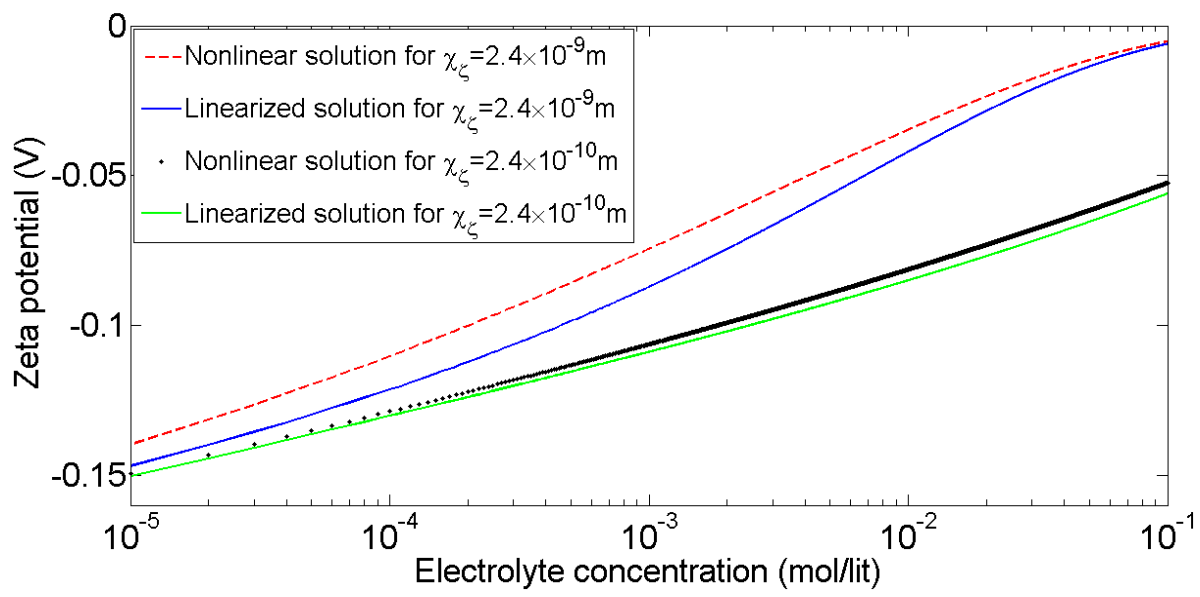

Figure 3. The variations of the zeta potential with electrolyte concentration using the solutions of the linearized and nonlinear PB equation for $\chi_{\zeta}=2.4 \times 10^{-9} \mathrm{~m}$ and $\chi_{\zeta}=2.4 \times 10^{-10} \mathrm{~m}$.

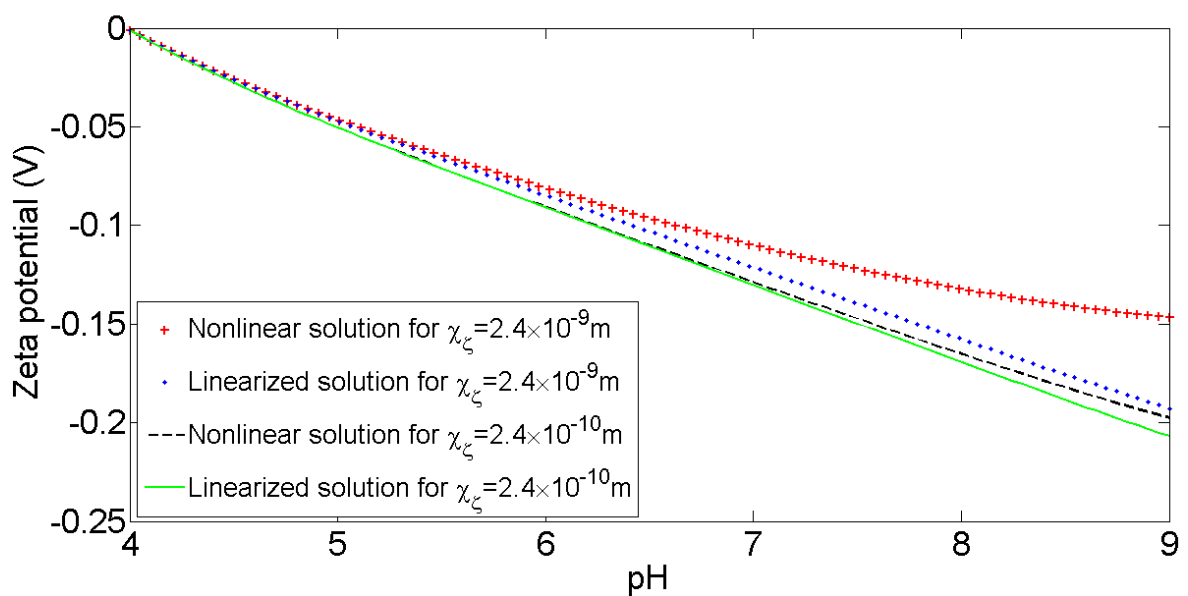

Figure 4. The variations of the zeta potential with fluid $\mathrm{pH}$ using the solutions of the linearized and nonlinear PB equation for $\chi_{\zeta}=2.4 \times 10^{-9} \mathrm{~m}$ and $\chi_{\zeta}=2.4 \times 10^{-10} \mathrm{~m}\left(C_{b}=10^{-4} \mathrm{M}\right)$.

The variations of the zeta potential with fluid $\mathrm{pH}$ are also predicted using the linearized and nonlinear solutions at $C_{b}=10^{-4} \mathrm{M}$ as shown in Fig. 4. The results show that the zeta potential in magnitude increases with increasing fluid $\mathrm{pH}$ as reported in 28 . Besides that, the difference of the zeta potential between the linearized and nonlinear $\mathrm{PB}$ solutions increases with increasing fluid $\mathrm{pH}$. For fluid $\mathrm{pH}=5-8$ that is normally encountered in published data and $\chi_{\zeta}=2.4 \times 10^{-10} \mathrm{~m}$, the values of the zeta potential predicted from the linearized and nonlinear solutions are almost the same. 


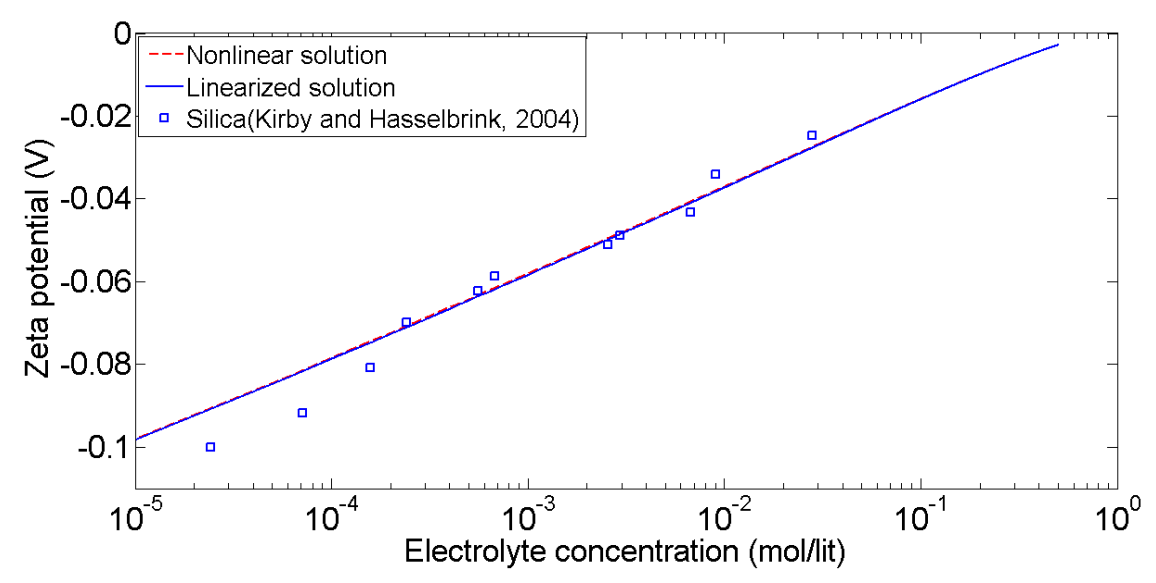

(a) $T=22.6^{\circ} \mathrm{C}, \mathrm{pH}=7, \mathrm{~K}_{\mathrm{Me}}=10^{-7.5}, K_{(-)}=10^{-8.4}, \Gamma_{S}^{0}=10 \times 10^{18}$ site $/ \mathrm{m}^{2}$ and $\chi_{\zeta}=2.4 \times 10^{-10} \mathrm{~m}$ (Experimental data obtained from Kirby and Hasselbrink, 2004 [28])

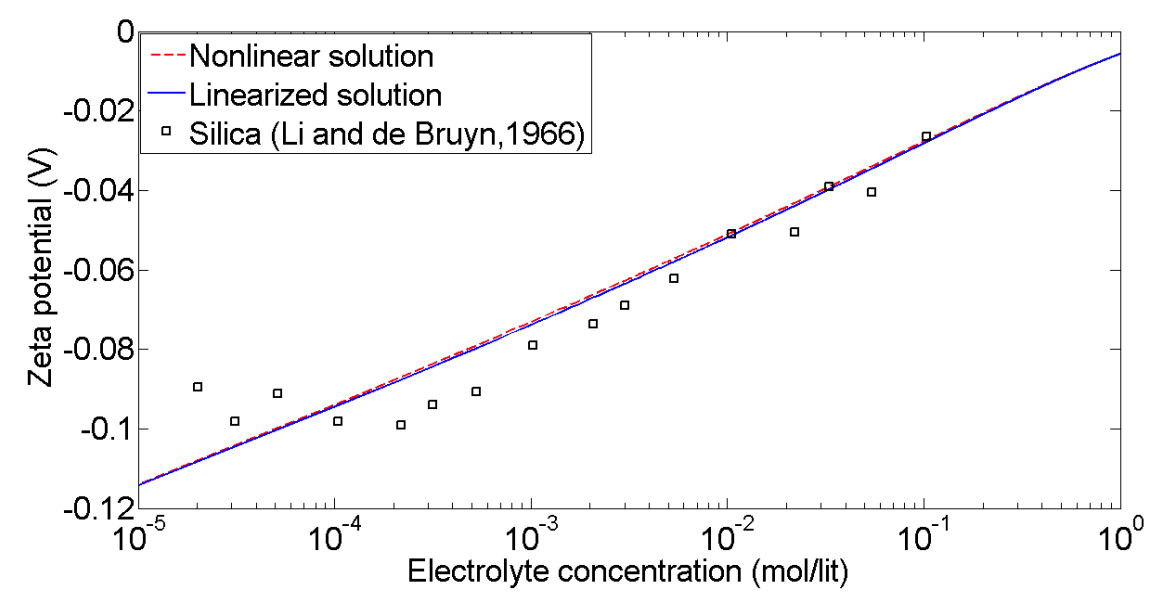

(b) $T=23^{\circ} \mathrm{C}, \mathrm{pH}=7, \mathrm{~K}_{\mathrm{Me}}=10^{-7.5}, K_{(-)}=10^{-8.0}, \Gamma_{S}^{0}=10 \times 10^{18} \mathrm{site} / \mathrm{m}^{2}$ and $\chi_{\zeta}=2.4 \times 10^{-10} \mathrm{~m}$ (Experimental data obtained from Li and de Bruyn, 1966 [29]).

Figure 5. The zeta potential as a function of electrolyte concentration compared with experimental data from $[28,29]$ for silica.

Glover et al. [15] have compared the zeta potential as a function of electrolyte concentration predicted from the linearized solution to published experimental data for silica. They used the input parameters for modeling as: (1) $T=22.6^{\circ} \mathrm{C}, \mathrm{pH}=7, \mathrm{~K}_{\mathrm{Me}}=10^{-7.5}, K_{(-)}=10^{-8.4}, \Gamma_{S}^{0}=10 \times 10^{18} \mathrm{site} / \mathrm{m}^{2}$ and $\chi_{\zeta}=2.4 \times 10^{-10} \mathrm{~m}$ for the experimental data obtained from [28] (see Fig. 5a); (2) $T=23^{\circ} \mathrm{C}, \mathrm{pH}=$ $7, K_{\mathrm{Me}}=10^{-7.5}, K_{(-)}=10^{-8.0}, \Gamma_{S}^{0}=10 \times 10^{18}$ site $/ \mathrm{m}^{2}$ and $\chi_{\zeta}=2.4 \times 10^{-10} \mathrm{~m}$ for the experimental data obtained from [29] (see Fig. 5b). The same input parameters are used in this work to compare the 
experimental data to those predicted from the nonlinear solution (see the dashed lines in Fig. 5). It is seen that the theoretical results obtained from the linearized and nonlinear solutions are in very good agreement with each other for $\mathrm{pH}=7$ and $\chi_{\zeta}=2.4 \times 10^{-10} \mathrm{~m}$ as stated above. Additionally, they are also in agreement with the general trend in the experimentally derived zeta-potential values from [28, 29] (see symbols).

\section{Conclusions}

The comparison between the linearized and nonlinear PB solutions has been performed for silica surfaces. It is found that the linearized solution always overestimates the absolute value of the electric potential in the EDL as well as the zeta potential. For a small magnitude of the surface potential $\left(\left|\varphi_{d}\right| \leq 25 \mathrm{mV}\right)$, the linearized PB solution could be used to predict the electric potential distribution in the EDL instead of the more complicated nonlinear PB solution. It is also shown that the degree of deviation between the linearized and nonlinear solution in determining the zeta potential strongly depends on electrolyte concentration, fluid $\mathrm{pH}$ and shear plane distance. At a given electrolyte concentration, the zeta potential computed from the linearized PB solution closely matches with that computed from the nonlinear solution for the fluid $\mathrm{pH}=5-8$ that is normally encountered in published experimental data and the shear plane distance of $2.4 \times 10^{-10} \mathrm{~m}$. Therefore, the solution of linearized PB equation can be used to calculate the zeta potential under that condition. This is validated by comparing the linearized and nonlinear solutions with experimental data in literature. In particularly, if the shear distance is taken as $2.4 \times 10^{-9} \mathrm{~m}$ and the fluid $\mathrm{pH}$ is larger than 8 , the nonlinear PB solution needs to be taken into account to calculate the zeta potential.

\section{References}

[1] Jouniaux, L., and T. Ishido (2012), Electrokinetics in earth sciences: A tutorial, International Journal of Geophysics, 2012, Article ID 286107.

[2] Wurmstich, B., and F. D. Morgan (1994), Modeling of streaming potential responses caused by oil well pumping, Geophysics, 59(1), 46-56.

[3] Ishido, T., and J. Pritchett (1999), Numerical simulation of electrokinetic potentials associated with subsurface fluid flow, Journal of Geophysical Research, 104(B7), 5,24715,259.

[4] Jouniaux, L., J. Pozzi, J. Berthier, and P. Masse' (1999), Detection of fluid flow variations at the nankai trough by electric and magnetic measurements in boreholes or at the seafloor, Journal of Geophysical Research, 104(B12), 29,29329,309.

[5] Titov, K., A. Revil, P. Konosavsky, S. Straface, and S. Troisi (2005), Numerical modeling of self potential signals associated with a pumping test experiment, Geophysical Journal International, 162(2), 641-650.

[6] Aizawa, K., Y. Ogawa, and T. Ishido (2009), Groundwater flow and hydrothermal systems within volcanic edifices: Delineation by electric self-potential and magnetotellurics, Journal of Geophysical Research, 114(B01208).

[7] Corwin, R. F., and D. B. Hoover (1979), The self-potential method in geothermal exploration, Geophysics, 44(2), 226-245.

[8] Morgan, F. D., E. R. Williams, and T. R. Madden (1989), Streaming potential properties of westerly granite with applications, Journal of Geophysical Research, 94(B9), 12.449-12.461.

[9] Saracco, G., P. Labazuy, and F. Moreau (2004), Localization of self-potential sources in volcano electric effect with complex continuous wavelet transform and electrical tomography methods for an active volcano, Geophysical Research Letters, 31, L12619, doi:10.1029/2004GL019,554. 
[10] Martinez-Pagan, P., A. Jardani, A. Revil, and A. Haas (2010), Self-potential monitoring of a salt plume, geophysics, 75(4), WA17-WA25, doi:10.1190/1.3475533.

[11] Naudet, V., A. Revil, J.-Y. Bottero, and P. Bgassat (2003), Relationship between self potential (sp) signals and redox conditions in contaminated groundwater, Geophysical Research Letters, 30(21), doi:10.1029/2003GL018096.

[12] Saunders, J. H., M. D. Jackson, and C. C. Pain (2008), Fluid flow monitoring in oil fields using downhole measurements of electrokinetic potential, Geophysics, 73(5)(5), E165-E180.

[13] Mizutani, H., T. Ishido, T. Yokokura, and S. Ohnishi (1976), Electrokinetic phenomena associated with earthquakes, Geophysical Research Letters, 3(2).

[14] Revil, A., P. A. Pezard, and P. W. J. Glover (1999), Streaming potential in porous media 1. theory of the zeta potential, Journal of Geophysical Research, 104(B9), 20,021-20,031.

[15] Glover, P. W. J., E. Walker, and M. Jackson (2012), Streaming-potential coefficient of reservoir rock: A theoretical model, Geophysics, 77(2), D17-D43.

[16] Van Olphen, H. (1977), An Introduction to Clay and Colloidal Chemistry, Wiley Interscience, New York.

[17] Hunter, R. J. (1981), Zeta Potential in Colloid Science, Academic, New York.

[18] Behrens S. H. and D. G. Grier (2001), The charge of glass and silica surfaces, The Journal of Chemical Physics $115,6716-6721$

[19] Mitchell, J.K. (1976), Fundamentals of Soil Behavior, John Wiley \& Sons, Inc., New York.

[20] Mahanta, K.K., Mishra, G.C., Kansal, M.L. (2012), Estimation of electric double layer thickness from linearized and nonlinear solutions of Poisson-Boltzman equation for single type of ions, Appl. Clay Sci. 59-60, 1-7.

[21] Bard, Allen J., Faulkner, Larry R. (2001), Electrochemical Methods, Fundamentals and Applications, second ed. John Wiley and Sons, Inc.

[22] Butt, Hans-Jurgen, Graf, Karlheinz (2003), Physics and Chemistry of Interfaces, Wiley-VCH, Weinheim.

[23] Rice, C., and R. Whitehead (1965), Electrokinetic flow in a narrow cylindrical capillary, J.Phys. Chem., 69(11), 4017-4024.

[24] Pride, S. (1994), Governing equations for the coupled electromagnetics and acoustics of porous media, Physical Review B, 50(21), 15,678-15,696.

[25] Chapman, L., (1913), A contribution to the theory of electrocapillarity, Philosophical Magazine Series 625 (148), 475-481.

[26] Appelo, C.A.J., Postma, D., (2005), Geochemistry, Groundwater and Pollution, second ed. A. Balkema Publishers, Leiden/London/New York/Philadelphia/Singapore.

[27] Ishido, T., and H. Mizutani (1981), Experimental and theoretical basis of electrokinetic phenomena in rock-water systems and its applications to geophysics, Journal of Geophysical Research B, 86, 1763-1775.

[28] Kirby, B. J., and E. F. Hasselbrink (2004), Zeta potential of microfluidic substrates. 1. Theory, experimental techniques, and effects on separations, Electrophoresis, 25, 187-202.

[29] Li, H. C., and P. L. de Bruyn (1966), Electrokinetic and adsorption studies on quartz: Surface Science, 5, 203220. 